\title{
Isolated Nephron Segments from Rabbit Models of Obstructive Nephropathy
}

\author{
Michael J. Hanley and Karen Davidson, Department of Medicine, Division of \\ Renal Diseases, University of Texas Health Science Center, \\ San Antonio, Texas 78284
}

A B S TRACT Micropuncture and microcatheterization studies have been used extensively to investigate the pathophysiologic alterations in renal function induced by urinary tract obstruction. The present isolated tubule microperfusion studies were designed to examine the intrinsic alterations in segmental nephron function induced by $24 \mathrm{~h}$ of bilateral (BUO) and unilateral (UUO) urinary tract obstruction.

Following UUO superficial proximal convoluted tubule reabsorption rate $\left(\mathrm{J}_{\mathrm{v}}\right)$ was not different from contralateral control $(0.75 \pm 0.08$ vs. $0.73 \pm 0.11 \mathrm{nl} / \mathrm{mm}$ per min, NS). Following UUO $\mathrm{J}_{\mathrm{v}}$ in juxtamedullary proximal convoluted tubules (JMPCT) was reduced $32 \%$ $(0.69 \pm 0.06$ vs. $0.47 \pm 0.04 \mathrm{nl} / \mathrm{mm}$ per $\min , P<0.02)$. Following UUO $\mathrm{J}_{\mathrm{v}}$ in proximal straight tubules (PST) was reduced $52 \%(0.25 \pm 0.02$ vs. $0.12 \pm 0.03, P<0.01)$. Thick ascending limb (T-ALH) function was assessed by measurement of ability to lower perfusate chloride ion concentration $(\triangle \mathrm{Cl})$. Following $\mathrm{UUO} \Delta \mathrm{Cl}$ was reduced $76 \%(-39 \pm 9$ vs. $-9 \pm 1 \mathrm{meq} / \mathrm{liter}, P<0.001)$. Cortical collecting tubule (CCT) function was assessed by measurement of antiduiretic hormone (ADH)-dependent osmotic water flow. Following UUO osmotic water flow was reduced $76 \%(0.90 \pm 0.08$ vs. $0.22 \pm 0.04$ $\mathrm{nl} / \mathrm{mm}$ per $\min , P<0.01)$ and this $\mathrm{ADH}$ resistance could not be overcome by cAMP. Nephron segments were then examined following relief of BUO. There were no differences in intrinsic function following relief of BUO when compared with UUO. We conclude that in UUO and BUO $(a)$ the intrinsic tubular defects are identical, $(b)$ the natriuresis noted is due, in part, to disordered JMPCT, PST, and T-ALH NaCl reabsorption, $(c)$ the impaired concentrating ability is due,

Portions of this work were presented at the 13th Annual Meeting of the American Society of Nephrology, Wash., D. C., November 23, 1980, and at the 38th Annual Meeting of the American Federation for Clinical Research, San Francisco, April 26, 1981.

Received for publication 4 May 1981 and in revised form 21 September 1981. in part, to depressed function in T-ALH and ADH resistance of the CCT, and $(d)$ the ADH resistance occurs at a site distal to the intracellular generation of cAMP.

\section{INTRODUCTION}

As has been demonstrated in many studies in man and experimental animals, urinary tract obstruction induces pathological changes in renal function $(1,2)$. The character and magnitude of these changes appear to depend on whether the obstruction involves one or both kidneys. After release of bilateral ureteral obstruction a diuresis and natriuresis usually occurs that is not observed after release of unilateral obstruction. During the postobstructive diuresis following relief of bilateral obstruction, surface micropuncture studies indicate that proximal tubule reabsorption is normal or reduced while distal tubule reabsorption is consistently reduced $(3,4)$. Papillary micropuncture studies demonstrate a reduction in proximal reabsorption of deep nephrons before the bend in Henle's loop and normal functioning of the terminal papillary collecting duct (5). Evaluation of medullary collecting duct function following relief of bilateral ureteral obstruction by the microcatheterization technique showed no significant reabsorption of salt and water along the length of this segment (6). After release of unilateral ureteral obstruction, micropuncture studies indicate that there is increased fractional reabsorption in proximal and distal tubules of surface nephrons $(7,8)$. Papillary micropuncture in this setting again shows a decreased fractional sodium reabsorption before the bend of Henle's loop of deep nephrons and a normal functioning of the papillary collecting duct $(9,10)$. Microcatheterization of the medullary collecting duct following release of unilateral ureteral obstruction again demonstrated the same defect in collecting duct function as observed in bilateral obstruction.

Studies of bilateral and unilateral obstruction using 
clearance, micropuncture, or microcatheterization techniques do not provide a clear assessment of the direct effects of obstruction on intrinsic tubular functions. In these models, changes in tubular reabsorption rates are also critically dependent on volume status, possible circulating natriuretic factors as well as regional renal blood flow and its effect on interstitial composition and physical factors. Therefore, differences in multiple factors could be responsible for the postobstructive diuresis observed following relief of bilateral obstruction but not after release of unilateral obstruction. There have been studies examining the direct effect of in vitro increases in hydrostatic pressure on tubular function in isolated perfused tubules. They demonstrated that an acute in vitro increase in hydrostatic pressure has no effect on proximal convoluted tubule fluid reabsorption (11) but may alter transport in the cortical collecting tubule since the potential difference fell in response to this maneuver (12). The lack of information on intrinsic tubular function following relief of obstruction prompted us to develop a model of unilateral ureteral obstruction (UUO) and bilateral ureteral obstruction (BUO) for 24 $h$ in the New Zealand White rabbit (13). Following characterization of this model by clearance techniques the effect of $24 \mathrm{~h}$ of obstruction on intrinsic nephron function was examined by isolating segments from these obstructed kidneys and determining transport properties by the method of isolated tubule microperfusion. This not only allowed us to determine the effect of $24 \mathrm{~h}$ of obstruction on intrinsic segmental nephron function, but also allowed us to directly compare segments from unilaterally and bilaterally obstructed kidneys. In addition, we were able to examine segments not directly available to surface and papillary micropuncture or papillary microcatheterization.

\section{GLOSSARY}

$\begin{array}{ll}\text { ADH } & \text { Antidiuretic hormone } \\ \text { BUO } & \text { Bilateral ureteral obstruction } \\ \text { CCT } & \text { Cortical collecting tubule } \\ \Delta C l & \text { Chloride ion concentration, meq/liter } \\ \text { Cl Phe S-cAMP } & \text { 8-p-Cl-phenylthio-cAMP } \\ \text { Fe } \text { Na } & \text { Fractional excretion of sodium, \% } \\ \text { GFR } & \text { Glomerular filtration rate } \\ J_{v} & \text { Net fluid reabsorption, } n l / m m \text { per min } \\ \text { JMPCT } & \text { Juxtamedullary proximal convoluted tu- } \\ & \text { bule } \\ \text { PD } & \text { Potential difference, } m \text { V } \\ \text { P }_{\mathrm{f}} & \text { Transepithelial osmotic water permeabil- } \\ & \text { ity coefficient } \\ \text { PST } & \text { Cortical proximal straight tubule } \\ \text { SNGFR } & \text { Single nephron glomerular filtration rate } \\ \text { SPCT } & \text { Superficial proximal convoluted tubule } \\ \text { T-ALH } & \text { Cortical thick ascending limb of Henle's } \\ & \text { loop } \\ \text { UK } & \text { Unobstructed kidney }\end{array}$

$\begin{array}{ll}\mathrm{U}_{\mathrm{Na}} \mathrm{V} & \begin{array}{c}\text { Whole kidney excretion rate of sodium, } \\ \mu e q / \min \end{array} \\ \mathrm{UUO} & \text { Unilateral ureteral obstruction } \\ \mathrm{V} & \text { Tubule fluid flow rate, } \mathrm{nl} / \mathrm{min} \\ \overline{\mathrm{V}} & \text { Partial molar volume of water } \\ \mathrm{V}_{\mathrm{i}} & \text { Perfusion rate } \\ \mathrm{V}_{\mathrm{o}} & \text { Collection rate }\end{array}$

\section{METHODS}

Female New Zealand rabbits weighing 1.5-2.5 kg were used in all experiments. All rabbits were fed a standard laboratory diet and had free access to water. Studies were performed to assess the degree and pattern of renal abnormalities produced by $24 \mathrm{~h}$ of complete unilateral and bilateral ureteral obstruction. These animals were anesthetized with intravenous sodium methohexital (Brevital sodium, Eli Lilly, \& Co., Indianapolis, Ind.) and complete UNO or BUO was accomplished with sterile procedure by tying a 3-0 silk suture around the ureter(s) just above the ureterovesical junction. The animals were allowed to recover from anesthesia and were returned to individual cages where they were then given free access to food and water. $24 \mathrm{~h}$ later the animals were reanesthetized with intravenous barbiturate and one of the following two protocols was followed: $(a)$ standard clearance studies were performed immediately after relief of obstruction by catheterizing each ureter and obtaining separate urine collections. A 30 -min equilibration period of moderate volume expansion ( $5 \%$ of body wt) was followed by four timed 10-min urine collections during a constant infusion of normal saline containing inulin and pitressin or (b) the obstructed kidney was removed through a flank incision. The kidney was then sliced for dissection and microperfusion. The experimental UUO or BUO kidney is compared with the control unobstructed kidney (UK) in both clearance and microperfusion studies.

Segments of renal tubules were perfused in vitro by techniques previously described (14-16). After being removed, the kidney was cut into $1-2-\mathrm{mm}$ slices and the appropriate segment was dissected free in a chilled dish of artificial ultrafiltrate with $5 \% \mathrm{vol} / \mathrm{vol}$ fetal calf serum. The tubules were perfused with an artificial ultrafiltrate of the following composition (in $\mathrm{mM}$ ): $\mathrm{NaCl}, 105 ; \mathrm{KCl}, 5 ; \mathrm{NaHCO}_{3}, 25 ; \mathrm{Na}_{2} \mathrm{HPO}_{4}$, 2.3; sodium acetate, $10 ; \mathrm{MgSO}_{4}, 1 ; \mathrm{CaCl}_{2}, 1.8$; glucose, 8.3 , and alanine, 5 . The tubules were bathed with a solution of identical composition to which $5 \% \mathrm{vol} / \mathrm{vol}$ fetal calf serum had been added. In cortical collecting tubule (CCT) experiments in which an osmotic gradient was necessary, the bathing solution was identical in composition but the osmolality was increased $100 \mathrm{mosmol} / \mathrm{kg} \mathrm{H}_{2} \mathrm{O}$ ) by the addition of raffinose. All solutions were equilibrated with $95 \% \mathrm{O}_{2}-5 \% \mathrm{CO}_{2}$ and all experiments were performed at $37^{\circ} \mathrm{C}$. [ methoxy ${ }^{3} \mathrm{H}$ ] Inulin (NET-086L, New England Nuclear, Boston, Mass.) was exhaustively dialyzed and added to the perfusate as an impermeant volume marker in all tubules studied (17). To study uniform populations of tubules the following criteria were established: (a) segments of proximal convoluted tubules were selected from superficial or juxtamedullary nephrons; (b) the segments of cortical proximal straight tubules (PST) studies were from superficial nephrons and had their origin within 1-2 $\mathrm{mm}$ of the renal capsule; (the study of juxtamedullary PST in these models was not technically feasible) (c) segments of thick ascending limb of Henle's loops (T-ALH) were cortical in origin; and $(d)$ segments of collecting tubule were cortical in all cases. Fluid reabsorption $\left(J_{v}\right)$ in nanoliters per millimeter per minute was calculated 
by the following equation (11): $J_{v}=V_{i}-V_{o} / L$. The perfusion rate $\left(V_{i}\right)$ is calculated by dividing the total $\left[{ }^{3} \mathrm{H}\right]$ inulin counts per minute of the collected fluid by the $\left[{ }^{3} \mathrm{H}\right]$ inulin counts per minute per nanoliter of perfusion fluid and by the time of the collection period; the collection rate $\left(V_{0}\right)$ is obtained directly by a previously calibrated constant-volume pipette (18) and the time of the collection period; and $L$ is the length of the tubule in millimeters. In all segments, epithelial integrity was evaluated by visual inspection and bath sampling. The leakage rate of the $\left[{ }^{3} \mathrm{H}\right]$ inulin volume marker into the bath was $<1 \%$ of the perfusion rate (17). Potential difference (PD) was measured in all tubules by methods previously described (16). Because of the low flow rates used in the T-ALH, the perfusion rate was calculated by direct measurement of the collected fluid volume in calibrated micropipettes divided by the time of collection. Volume reabsorption was obtained in this segment by comparing the $\left[{ }^{3} \mathrm{H}\right]$ inulin counts per nanoliter of collected fluid to that of the perfusate. No significant volume reabsorption occurred in this segment in either control or obstructed tubules. Samples for chloride determinations were collected under oil. The PD was measured in T-ALH during perfusion and during stop flow conditions.

The functional capacity of the CCT was tested by measurement of fluid reabsorption in response to an abrupt increase $\left(100 \mathrm{mosmol} / \mathrm{kg} \mathrm{H}_{2} \mathrm{O}\right)$ in bath osmolality with raffinose in the presence of a maximum ADH stimulus $(250 \mu \mathrm{U} /$ $\mathrm{ml}$ ). In tubules from obstructed kidneys the measured change in inulin concentration correlated with the measured osmolality increase indicating tubule impermeability to raffinose or other solutes. Segments of CCT were equilibrated for $90 \mathrm{~min}$ before ADH $(250 \mu \mathrm{U} / \mathrm{ml})$ was added to the bathing solution. Sample collections were begun $10 \mathrm{~min}$ after the addition of ADH and continued for 30-60 min. (12). In CCT studies serial samples were collected for the measurement of fluid reabsorption and fluid osmolality. The transepithelial osmotic water permeability coefficient, $P_{f}$ (centimeters per second), was computed according to the expression derived by Al-Zahid and co-workers (17):

$$
P_{f}=-\frac{V_{i} C_{o}}{A \bar{V}_{w}}\left[\frac{C_{o}-C_{L}}{C_{o} C_{b} C_{L}}+\frac{1}{\left(C_{b}\right)^{2}} \operatorname{In} \frac{\left(C_{L}-C_{b}\right) C_{o}}{\left(C_{o}-C_{b}\right) C_{L}}\right]
$$

where $V_{i}$ is the perfusion rate; $C_{o}, C_{b}$, and $C_{L}$ are the osmolalities of the perfusate, bath, and collected fluids, respectively; $A$ is the luminal surface area; and $\bar{V}$ is the partial molar volume of water.

The time intervals between kidney removal and tubular perfusion were comparable for control and experimental segments. These times did not exceed $30 \mathrm{~min}$. for superficial proximal convoluted tubule (SPCT), juxtamedullary proxi- mal convoluted tubule (JMPCT), PST, and $1 \mathrm{~h}$ for CCT and T-ALH. In all studies, there were no statistical differences in either the perfusion rate or length between the control and experimental tubules. Osmolality of all solutions was determined by an osmometer (Advanced Instruments, Inc., Needham Heights, Mass.). Osmolality of collected fluid was determined by a modified Clifton nanoliter osmometer (Clifton Technical Physics, Hartford, N. Y.) (19). The analysis of chloride ion concentrations $(\Delta \mathrm{Cl})$ in the collected fluid and perfusate was performed by the microcoulometric titration method of Ramsay et al. (20). The results are expressed as means $\pm S E$ of the number of tubules studied. The statistical comparisons were performed by paired or unpaired $t$ test as appropriate.

\section{RESULTS}

Clearance studies. To evaluate the models of UUO and BUO in the New Zealand White rabbit, standard clearance studies were performed following catheterization of each ureter. The results of these studies are presented in Table I. Glomerular filtration rate (GFR) is reduced $67 \%(7.63 \pm 1.45$ vs. $2.50 \pm 0.32 \mathrm{ml} / \mathrm{min}$, $P<0.01)$ and fractional excretion of sodium $\left(\mathrm{FE}_{\mathrm{Na}}\right)$ is significantly increased $(2.66 \pm 0.94$ vs. $8.07 \pm 1.57 \%$, $P<0.02)$ in the postobstructed UUO kidney when compared with the contralateral UK. Urine osmolality is also significantly reduced in the postobstructed UUO kidney $(581 \pm 70$ vs. $301 \pm 15 \mathrm{mosmol}, P<0.01)$ and this value was not different from the corresponding plasma osmolality (UUO plasma osmolality $=303 \pm 6$ ). Following release of BUO, GFR was not statistically different from that observed following release of UUO $(2.50 \pm 0.32$ vs. $2.41 \pm 0.24 \mathrm{ml} / \mathrm{min}$, NS) although there was a statistically significant increase in $\mathrm{FE}_{\mathrm{Na}}$ $(8.07 \pm 1.57$ vs. $23.4 \pm 1.8 \%, P<0.01)$. Compared with UUO, urine flow rate was increased $63 \%$ following release of BUO $(0.406 \pm 0.061$ vs. $0.662 \pm 0.055 \mathrm{ml} / \mathrm{min}$, $P<0.01$ ). As in the case of UUO, urine osmolality following release of BUO was not significantly different from plasma values in these animals (BUO plasma osmolality $=335 \pm 6$ ). These studies demonstrate that this model possesses the necessary features of obstructive nephropathy, i.e., decreased GFR, a concentrating

TABLE I

Renal Function following Release of 24 h Obstruction

\begin{tabular}{|c|c|c|c|c|c|}
\hline & GFR & $\mathrm{FE}_{\mathrm{Ne}}$ & $\mathrm{U}_{\mathrm{Na}} \mathrm{V}$ & $\mathrm{v}$ & Uosmol \\
\hline & $m l / m i n$ & $\%$ & $\mu e q / \min$ & $n l / \min$ & \\
\hline UK $(n=8)$ & $7.63 \pm 1.45$ & $2.66 \pm 0.94$ & $32.6 \pm 6.1$ & $0.333 \pm 0.056$ & $581 \pm 70$ \\
\hline UUO $(n=8)$ & $2.50 \pm 0.32$ & $8.07 \pm 1.57$ & $34.8 \pm 8.0$ & $0.406 \pm 0.061$ & $301 \pm 15$ \\
\hline$P$ (UUO vs. UK) & $<0.01$ & $<0.02$ & NS & NS & $<0.01$ \\
\hline BUO $(n=9)$ & $2.41 \pm 0.24$ & $23.4 \pm 1.8$ & $71.2 \pm 5.3$ & $0.662 \pm 0.055$ & $351 \pm 12$ \\
\hline$P$ (BUO vs. UUO) & NS & $<0.01$ & $<0.01$ & $<0.01$ & - \\
\hline
\end{tabular}

Uosmol, urine osmolality. 
TABLE II

Effect of $24 h$ of Obstruction on the SPCT

\begin{tabular}{|c|c|c|c|c|}
\hline & $\mathrm{J}_{\mathrm{v}}$ & PD & Length & Perfusion rate \\
\hline & $n l / m m / m i n$ & $m V$ & $m m$ & $n l / m i n$ \\
\hline UK $(n=4)$ & $0.75 \pm 0.08$ & $-2.0 \pm 0.2$ & $1.2 \pm 0.1$ & $12.49 \pm 0.56$ \\
\hline UUO $(n=6)$ & $0.73 \pm 0.11$ & $-0.9 \pm 0.2$ & $1.2 \pm 0.1$ & $13.32 \pm 0.43$ \\
\hline$P$ (UUO vs. UK) & NS & $<0.02$ & NS & NS \\
\hline BUO $(n=6)$ & $0.80 \pm 0.08$ & $-0.8 \pm 0.1$ & $1.2 \pm 0.1$ & $13.46 \pm 0.49$ \\
\hline$P$ (UUO vs. BUO) & NS & NS & NS & NS \\
\hline
\end{tabular}

defect, and high fractional sodium excretion. The qualitative differences in clearance measurements from previous studies $(5,7)$ reflect the moderate volume expansion before the clearance studies.

SPCT. Segments of SPCT were dissected, perfused, and measurements were made of $\mathrm{J}_{\mathrm{v}}$ and PD (Table II). Tubules were obtained from kidneys subjected to UUO, the contralateral UK, and kidneys from animals subjected to $24 \mathrm{~h}$ of BUO. $\mathrm{J}_{\mathrm{v}}$ in SPCT obtained from UUO kidneys was not significantly different from the rates observed in tubules from the UK $(0.73 \pm 0.11 \mathrm{vs}$. $0.80 \mathrm{nl} / \mathrm{mm}$ per min, NS). Furthermore, there was no difference between $J_{v}$ in tubules obtained from UUO and BUO kidneys $(0.73 \pm 0.11$ vs. $0.80 \pm 0.08 \mathrm{nl} / \mathrm{mm}$ per min, NS). Obstruction did produce a small change in the observed PD in tubules from UUO and BUO kidneys $(-2.0 \pm 0.2$ vs. $-0.9 \pm 0.2 \mathrm{mV}, P<0.02)$ but without an observable effect on reabsorption rates. Obstruction for $24 \mathrm{~h}$, whether unilateral or bilateral, did not significantly alter SPCT function following relief of UUO or BUO. No observable morphologic changes were noted in SPCT segments from UK, UUO, or BUO kidneys.

JMPCT. Segments of JMPCT were dissected, perfused, and measurements made of $\mathrm{J}_{\mathrm{v}}$ and PD (Table III). As in the SPCT, tubules were obtained from kidneys subjected to UUO, the contralateral UK, and kidneys from animals subjected to $24 \mathrm{~h}$ of BUO. $J_{v}$ in tubules obtained from UUO kidneys was decreased $32 \%$ from control values (UK) $(0.69 \pm 0.06$ vs. $0.47 \pm 0.04$ $\mathrm{nl} / \mathrm{mm}$ per $\min , P<0.02)$. $\mathrm{J}_{\mathrm{v}}$ in tubules obtained from BUO kidneys was not significantly different from that in UUO kidneys $(0.47 \pm 0.04$ vs. $0.46 \pm 0.06 \mathrm{nl} / \mathrm{min}$ per min, NS). There were no statistical differences in the PD between the different groups (UK, UUO, BUO). Obstruction for $24 \mathrm{~h}$, whether unilateral or bilateral, does compromise function in the JMPCT. No observable morphologic changes were noted in JMPCT segments from UK, UUO, or BUO kidneys.

PST. Segments of PST were dissected, perfused, and measurements made of $\mathrm{J}_{\mathrm{v}}$ and PD (Table IV). Tubules were obtained from kidneys subjected to UUO, the contralateral UK, and kidneys from animals subjected to $24 \mathrm{~h}$ of BUO. $\mathrm{J}_{\mathrm{v}}$ in tubules obtained from UUO kidneys was decreased $52 \%$ from control values (UK) $(0.25 \pm 0.02$ vs. $0.12 \pm 0.03 \mathrm{nl} / \mathrm{mm}$, per min, $P$ $<0.01$ ). $\mathrm{J}_{\mathrm{v}}$ in tubules obtained from BUO kidneys was not significantly different from that in UUO kidneys $(0.12 \pm 0.03$ vs. $0.16 \pm 0.02 \mathrm{nl} / \mathrm{mm}$ per min, NS). Obstruction did produce a small change in the observed $P D$ in tubules from UUO kidneys $(-2.0 \pm 0.5$ vs. $-0.8 \pm 0.2 \mathrm{mV}, P<0.02)$. No observable morphologic changes were noted in PST segments from UK, UUO, or BUO kidneys.

$T-A L H$. Tubules were obtained from kidneys subjected to UUO, the contralateral UK, and kidneys from animals subjected to $24 \mathrm{~h}$ of BUO. The functional integrity of this segment was assessed by measurement of the ability to lower the perfusate chloride ion concentration. Segments of T-ALH were dissected, per-

TABLE III

Effect of $24 h$ of Obstruction on the JMPCT

\begin{tabular}{lcccc}
\hline & Jv & PD & Length & Perfusion rate \\
\hline & $n l / m m / m i n$ & $m V$ & $m m$ & $n l / m i n$ \\
UK $(n=7)$ & $0.69 \pm 0.06$ & $-2.0 \pm 0.5$ & $1.0 \pm 0.1$ & $13.54 \pm 0.60$ \\
UUO $(n=6)$ & $0.47 \pm 0.04$ & $-1.0 \pm 0.5$ & $1.1 \pm 0.1$ & $13.42 \pm 0.43$ \\
$P($ UO vs. UK) & $<0.02$ & NS & NS & NS \\
BUO $(n=7)$ & $0.46 \pm 0.06$ & $0.8 \pm 0.1$ & $1.1 \pm 0.1$ & $13.25 \pm 0.34$ \\
$P($ UUO vs. BUO) & NS & NS & NS & NS \\
\hline
\end{tabular}


TABLE IV

Effect of $24 h$ of Obstruction on the PST

\begin{tabular}{lcccc}
\hline & Jv & PD & Length & Perfusion rate \\
\hline & $n l / m m / m i n$ & $m V$ & $m m$ & $n l / m i n$ \\
UK $(n=6)$ & $0.25 \pm 0.02$ & $-2.0 \pm 0.5$ & $1.73 \pm 0.09$ & $12.91 \pm 0.33$ \\
UUO $(n=6)$ & $0.12 \pm 0.03$ & $-0.8 \pm 0.2$ & $1.63 \pm 0.15$ & $12.88 \pm 0.20$ \\
$P($ UUO vs. UK) & $<0.01$ & $<0.02$ & NS & NS \\
BUO $(n=7)$ & $0.16 \pm 0.02$ & $-1.4 \pm 0.3$ & $1.49 \pm 0.07$ & $15.16 \pm 0.63$ \\
$P($ UUO vs. BUO) & NS & NS & NS & $<0.01$ \\
\hline
\end{tabular}

fused, and the fall in collected fluid chloride concentration was measured (Table V). Tubules obtained from animals that had undergone UUO or BUO appeared to have a distended lumen during dissection as opposed to tubules from normal rabbit kidneys and UK, which are generally completely collapsed obliterating the tubule lumen. Following UUO the ability of the T-ALH to lower the perfusion solution chloride ion concentration is reduced $76 \%(-39 \pm 9$ vs. $-9 \pm 1$ meq/liter, $P<0.01$ ). There was no significant difference between UUO and UK PD, perfusion rate, or length. Following relief of BUO the ability of the TALH to lower the perfusion solution chloride ion concentration was not different from that determined following relief of UUO $(-10 \pm 1$ vs. $-9 \pm 1 \mathrm{meq} /$ liter, NS). Both BUO and UUO impaired the functional capacity of the T-ALH to remove $\mathrm{NaCl}$ from the luminal fluid; and they did so to a similar degree. Although the PD in tubules from obstructed kidneys was not significantly different from the PD in tubules from unobstructed kidneys, there was a significant difference in the maximum PD obtained during stop flow conditions $(\mathrm{UK}=+15 \pm 1 \mathrm{mV}$, UUO $=+5 \pm 1 \mathrm{mV}$, BUO $=+6 \pm 1$ $\mathrm{mV}, P<0.01)$ further indicating that a transport defect is present following obstruction. During perfusion no observable morphologic changes were noted in $\mathrm{T}$ ALH segments from obstructed kidneys.

CCT. $\mathrm{P}_{\mathrm{f}}$ was measured in the presence of a maximum ADH $(250 \mu \mathrm{U} / \mathrm{ml})$ and cAMP stimulus. In tubules from normal kidneys it has been previously shown (21) that adding $0.1 \mathrm{mM} 8-p$-Cl-phenylthiocyclic $3^{\prime}, 5^{\prime}$ adenosine monophosphate (Cl Phe S-cAMP) to the bath duplicates a maximum ADH stimulus. Since it was determined that the ADH and Cl Phe S-cAMP response of the CCT from control (UK) kidneys was not different from that observed previously in normal tubules in this laboratory (22), the data for $J_{v}$ and $P_{f}$ in control (UK) tubules with these agents was pooled. Tubules were obtained from kidneys subjected to UUO, the contralateral control kidneys (UK), and kidneys from animals subjected to $24 \mathrm{~h}$ of BUO for the determination of $J_{v}$ and $P_{f}($ Table VI). Tubules obtained from animals that had undergone UUO or BUO appeared normal during microdissection but with the onset of microperfusion clear intracellular vesicles were noted in some cells. Following relief of UUO the ability of the collecting duct to respond to $\mathrm{ADH}$ was reduced $76 \%(0.90 \pm 0.08$ vs. $0.22 \pm 0.04 \mathrm{nl} / \mathrm{mm}$ per min, $P<0.01$ ). Following relief of BUO the ability of the collecting duct to respond to ADH was not different from that determined following relief of UUO $(0.22 \pm 0.04$ vs. $0.23 \pm 0.04 \mathrm{nl} / \mathrm{mm}$ per min, NS). BUO and UUO equally impaired the ability of the CCT to respond to $\mathrm{ADH}$. $\mathrm{ADH}$ increases the water permeability of the CCT by a complex series of intracellular events beginning with the intracellular generation of cAMP. In order to determine if the obstruction-induced defect in $\mathrm{ADH}$ response occurred before or after the generation of intracellular cAMP, a cell permeable cAMP was employed to increase water permeability

TABLE V

Effect of Obstruction on the T-ALH

\begin{tabular}{lcccc}
\hline & PD & $\Delta \mathrm{Cl}$ & $\begin{array}{c}\text { Perfusion } \\
\text { rate }\end{array}$ & Length \\
\hline & $m V$ & $m e q /$ liter & $n l / m i n$ & $m m$ \\
UK $(n=6)$ & $+5 \pm 1$ & $-37 \pm 3$ & $0.98 \pm 0.02$ & $1.1 \pm 0.1$ \\
UUO $(n=6)$ & $+4 \pm 1$ & $-9 \pm 1$ & $1.06 \pm 0.03$ & $1.2 \pm 0.1$ \\
$P($ UO vs. UK) & NS & $<.001$ & NS & NS \\
BUO $(n=7)$ & $+4 \pm 1$ & $-10 \pm 1$ & $1.05 \pm 0.03$ & $1.5 \pm 0.1$ \\
$P($ UUO vs. BUO) & NS & NS & NS & NS \\
\hline
\end{tabular}


TABLE VI

Effect of Obstruction on ADH-dependent Water Flow in the CCT

\begin{tabular}{|c|c|c|c|c|c|c|}
\hline & \multirow{2}{*}{$\begin{array}{c}\text { Control } \\
\text { PD }\end{array}$} & \multicolumn{2}{|c|}{$\mathrm{J}_{\mathrm{v}}$} & \multirow[b]{2}{*}{$\mathbf{P}_{\mathbf{1}}$} & \multirow[b]{2}{*}{ Perfusion rate } & \multirow[b]{2}{*}{ Length } \\
\hline & & Control & ADH & & & \\
\hline & $m V$ & \multicolumn{2}{|c|}{$n l / m m / m i n$} & $\mathrm{~cm} / \mathrm{s}$ & $n l / \min$ & $m m$ \\
\hline UK $(n=7)$ & $-10 \pm 2$ & $-0.04 \pm 0.01$ & $0.90 \pm 0.08$ & $0.0194 \pm 0.0045$ & $14.81 \pm 0.72$ & $1.40 \pm 0.10$ \\
\hline UUO $(n=6)$ & $+3 \pm 4$ & $0.00 \pm 0.03$ & $0.22 \pm 0.04$ & $0.0042 \pm 0.0010$ & $13.51 \pm 0.98$ & $1.20 \pm 0.20$ \\
\hline$P$ (UUO vs. UK) & $<0.02$ & - & $<0.01$ & $<0.02$ & NS & NS \\
\hline BUO $(n=7)$ & $-3 \pm 6$ & $-0.04 \pm 02$ & $0.23 \pm 0.04$ & $0.0030 \pm 0.0005$ & $15.16 \pm 0.28$ & $1.54 \pm 0.12$ \\
\hline$P$ (BUO vs. UUO) & NS & - & NS & NS & NS & NS \\
\hline
\end{tabular}

in tubules from UUO kidneys (Table VII). Cl Phe ScAMP-dependent water flow was significantly impaired $(0.90 \pm 0.08$ vs. $0.36 \pm 0.12 \mathrm{nl} / \mathrm{min}$ per $\min , P$ $<0.01)$ following relief of $24 \mathrm{~h}$ of obstruction. This suggests that the defect in ADH response induced by obstruction lies somewhere beyond the intracellular generation of cAMP.

\section{DISCUSSION}

Since ureteral obstruction results in a myriad of renal physiologic alterations, the direct effect of obstruction on the function of the renal tubules has been difficult to define. Previous studies in isolated tubule preparations $(11,12)$ have examined the effect of acutely increasing hydrostatic pressure on tubular transport. When hydrostatic pressure was increased in the proximal convoluted tubule, resulting in marked tubular dilatation, there was no significant change in fractional or absolute reabsorption while the perfusion rate was kept constant. A similar inference was made by these authors on the effect of hydrostatic pressure on PST function. Evaluation of the effect of acute pressure changes on distal nephron function have been more indirect. It is generally assumed that the normally negative luminal PD in the CCT is the result of active sodium transport. Increasing hydrostatic pressure in the CCT caused a fall in the negative luminal PD, independent of flow rate, implying a decrease in active sodium transport. A recent study (23) examining sodium transport isotopically found that a general correlation between sodium transport and PD did exist but this appeared to be highly variable. Thus, there is some suggestive evidence that obstruction does interfere with sodium reabsorption by the CCT. It has also been demonstrated (24) that the electrical resistance of the CCT was unchanged when hydrostatic pressure was increased even though PD was found to decrease. These studies also support the view that an acute hydrostatic pressure increase, acts to decrease active sodium transport by the CCT. In the present examination of obstructive nephropathy by isolated tubule microperfusion, tubules have been removed from obstructed kidneys and studied without in vivo reflow. The tacit assumption has been made that the in vitro microperfusion of these segments simulates those in vivo experimental studies that examined nephron function immediately after release of obstruction.

Following relief of UUO there is a decrease in GFR with a decrease in abolute sodium excretion and an increase in fractional sodium excretion. Micropuncture of surface nephrons in this setting has shown a $28 \%$ increase in fractional reabsorption in the proximal tubule with a 55\% decrease in absolute reabsorption (8).

TABLE VII

Effect of Obstruction on cAMP-dependent Water Flow in the CCT

\begin{tabular}{|c|c|c|c|c|c|c|}
\hline & \multirow[b]{2}{*}{$\begin{array}{c}\text { Control } \\
\text { PD }\end{array}$} & \multicolumn{2}{|c|}{$\mathbf{J}_{\mathbf{v}}$} & \multirow[b]{2}{*}{$\mathbf{P}_{\mathbf{f}}$} & \multirow[b]{2}{*}{ Perfusion rate } & \multirow[b]{2}{*}{ Length } \\
\hline & & Control & сAMP & & & \\
\hline & $m V$ & \multicolumn{2}{|c|}{$n l / m m / m i n$} & $\mathrm{~cm} / \mathrm{s}$ & $n l / \min$ & $m m$ \\
\hline UK $(n=7)$ & $-10 \pm 2$ & $-0.04 \pm 0.01$ & $0.90 \pm 0.08$ & $0.0194 \pm 0.0045$ & $14.81 \pm 0.72$ & $1.40 \pm 0.10$ \\
\hline UUO $(n=6)$ & $-8 \pm 5$ & $-0.01 \pm 0.02$ & $0.36 \pm 0.12$ & $0.0045 \pm 0.0066$ & $14.30 \pm 0.66$ & $1.3 \pm 0.10$ \\
\hline$P$ (UUO vs. UK) & NS & - & $<0.01$ & $<0.05$ & NS & NS \\
\hline
\end{tabular}


These changes in reabsorption were accompanied by tubule heterogeneity. One study using the modified Hansen's technique showed filtration in only $40 \%$ of surface nephrons and $12 \%$ of juxtamedullary nephrons (8) while another (9) showed a smaller difference of 41 and $33 \%$, respectively. Fractional reabsorption was also found to be increased in the superficial distal tubule but absolute reabsorption was decreased because of low GFR (7). Direct examination of the intrinsic transport capacity of SPCT in the present study (Table II and Fig. 1) shows that the reabsorption rate in this segment following $24 \mathrm{~h}$ of UUO is essentially normal and identical to the contralateral control kidney, implying that the decrease in absolute reabsorption of surface proximal tubules is purely a function of decreased GFR. Papillary micropuncture has shown that proximal delivery from deep nephrons to the bend of Henle's loop is increased 50\% following relief of UUO (9). In addition, there appears to be an inward redistribution of single nephron glomerular filtration rate (SNGFR) since deep nephron SNGFR was better preserved than surface nephron SNGFR. Direct evaluation of JMPCT and PST reabsorptive rates in this study demonstrated that obstruction itself affects the intrinsic transport capacity of these segments depressing their reabsorption rates 32 and $50 \%$, respectively ( $\mathrm{Ta}$ ble III, IV, and Fig. 1). The PST utilized in this study were identified as being from cortical nephrons in that they had their origin within 1-2 min of the renal capsule. The actual anatomic location of these segments is the midcortical region of the kidney. Therefore,

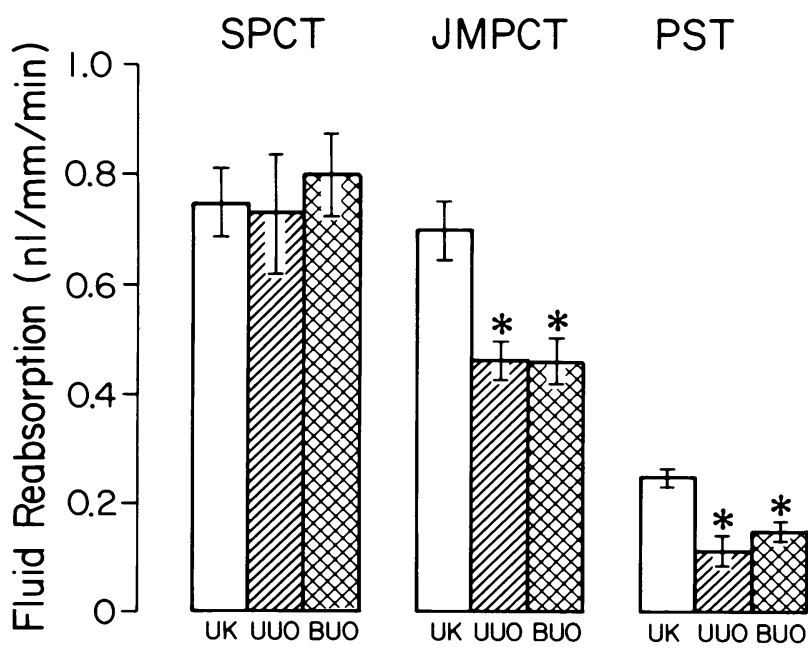

FIgURE 1 Effect of UUO and BUO on proximal nephron function. Clear bars represent the control unobstructed tubules, lined and hatched bars represent UUO and BUO tubules, respectively. Asterisk indicates differences from control tubules with $P<0.05$.

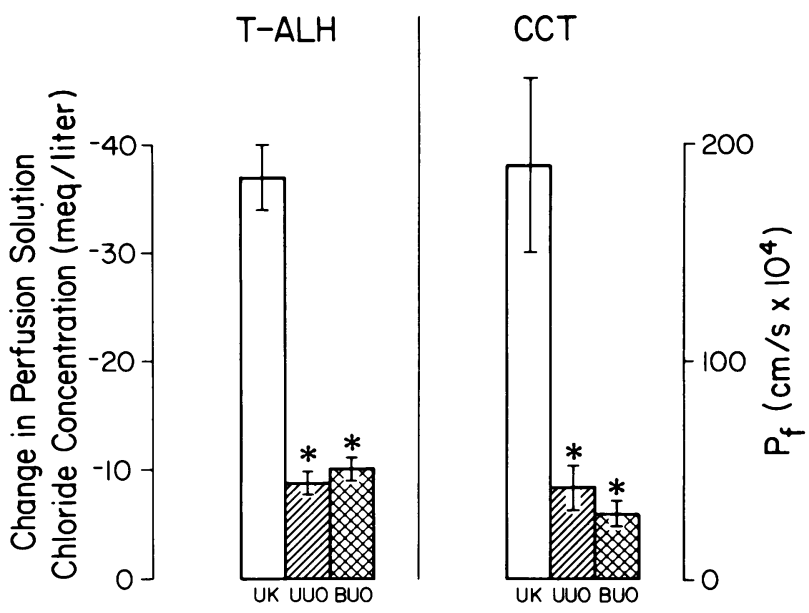

Figure 2. Effect of UUO and BUO on distal nephron function. Clear bars represent the control unobstructed tubules, lined and hatched bars represent UUO and BUO tubules, respectively. Asterisk indicates differences from control tubules with $P<0.05$.

these studies demonstrate that PST in the midcortical region and proximal convoluted tubules in the juxtamedullary region have depressed reabsorptive rates following obstruction. Although PST from juxtamedullary nephrons were not specifically examined it is likely that this segment was also adversely affected by the obstructive process. Increased delivery to the bend of the loop of deep nephrons (9) could be due to an isolated defect in JMPCT, or more likely to a defect in both the JMPCT and juxtamedullary PST.

Direct evaluation of T-ALH function following relief of UUO has not been possible using micropuncture techniques. Comparison of late proximal tubule and early distal tubule sodium delivery is difficult to interpret due to the low flow rates involved. Direct evaluation of the T-ALH following relief of UUO in the present study (Table V and Fig. 2) demonstrates significant functional impairment. This was suggested even during dissection, as these tubules appeared to be uncharacteristically dilated. This depression of TALH function could conceivably contribute to two observed phenomena following relief of obstruction. Decreased T-ALH function could contribute to the high sodium excretion as well as to the poor concentrating ability because of the dependence of medullary hypertonicity on T-ALH function.

The functional integrity of the collecting tubule is probably the most controversial aspect of obstructive pathophysiology. Microcatheterization data in the medullary collecting duct suggests that this segment no longer reabsorbs and may actually secrete salt and water following relief of obstruction (6). Micropunc- 
ture of the papillary collecting duct, on the other hand, indicates normal reabsorption of salt and water. Whether this difference is due to the segments examined or the technique employed is, at present, unclear. Sodium reabsorption was not specifically examined in this study because normal rates of sodium reabsorption in the CCT are low and highly variable when assessed by the technique of isolated tubule microperfusion. In the present study we examined the ability of the CCT to respond to ADH in the presence of an osmotic gradient. Following relief of obstruction isolated CCT demonstrated a significantly depressed ADH-dependent osmotic water permeability (Table VI and Fig. 2). We attempted to examine the mechanism involved in this defect by examining the response of tubules to a potent cAMP analog. The response to this agent was also significantly depressed suggesting that the defect induced by obstruction occurred at a site distal to the intracellular generation of cAMP (Table VII). These studies demonstrate that the concentrating defect following relief of UUO is due, in part, to ADH resistance of the CCT.

In all disease models examined thus far by the in vitro isolated tubule technique, an ADH resistance has been demonstrated in the CCT. In a study of the remnant model of chronic renal failure Fine et al. (26) examined the response of the CCT to ADH and a cAMP analogue (8-bromo cAMP). They observed a blunted hydroosmotic response to these agents, which was not further stimulated by theophylline (a phosphodiesterase inhibitor). An analysis of vasopressinstimulated adenyl cyclase activity in the remnant model by these investigators also demonstrated a $50 \%$ reduction with normal basal activity. From these studies they concluded that the ADH resistance of the CCT was due both to a defect in the tubule adenyl cyclase and to a defect beyond the generation of cAMP. Examination of the hydroosmotic ADH response in tubules from animals with ischemic acute renal failure (23) also demonstrates a significant defect. Whether all models examined thus far possess a defect in both adenyl cyclase and cellular response beyond the generation of cAMP has not been answered. The present studies suggest that following obstruction a defect is present beyond the intracellular generation of cAMP.

Following release of BUO, there is also a decrease in GFR and an increase in fractional sodium excretion. In contrast to UUO, tubular reabsorption in the proximal tubule of surface nephrons is normal (4) or even decreased (3). These same micropuncture studies show that reabsorption in the distal tubule is consistantly reduced. Direct examination of the intrinsic transport capacity of SPCT in the present study (Table II and Fig. 1) shows reabsorptive rates following relief of 24 $\mathrm{h}$ of BUO to be essentially normal and equal to that observed in UUO and in the UK. This would imply that any decrease in reabsorption following release of BUO was probably secondary to altered physical factors related to volume expansion or possible circulating natriuretic factors. Papillary micropuncture has demonstrated a $40 \%$ reduction in reabsorption before the bend of Henle's loop in deep nephrons (5). Direct evaluation of JMPCT and PST reabsorption rates in the present study (Table III, IV, and Fig. 2) shows that obstruction itself affects the intrinsic transport capacity of these segments depressing their reabsorption rates 32 and $36 \%$, respectively. As with UUO, although PST from juxtamedullary nephrons were not specifically examined, it is likely that this segment was adversely affected by the obstructive process. Increased delivery to the bend of the loop of deep nephrons after relief of BUO also could be due to an isolated defect in JMPCT or more likely to a defect in both JMPCT and juxtamedullary PST. Direct evaluation of T-ALH function following relief of BUO has not been possible using micropuncture techniques. Comparisons of end proximal and early distal tubule fluid-to-plasma (TF/ P) sodium and (TF/P) osmolality suggested that sodium and water reabsorption continued in the loop following relief of BUO but sodium reabsorption appeared to be impaired (4). Direct evaluation of T-ALH following relief of BUO in the present study (Table $\mathrm{V}$ and Fig. 2) demonstrates significant functional impairment. As in the UUO model, this was suggested even during dissection, because of the uncharacteristic tubular dilatation. Disordered T-ALH function in BUO could lead to the natriuresis and concentrating defect noted in this state. Microcatheterization of the medullary collecting tubule following relief of BUO suggests impaired reabsorption and even possible secretion of sodium and water (6). Papillary micropuncture following relief of BUO demonstrates intact functioning of the papillary collecting duct. The functional integrity of the CCT following relief of BUO in the present study was evaluated in a manner similar to that used following relief of UUO. Isolated CCT exhibited a significantly depressed response in ADHdependent osmotic water movement (Table VI and Fig. 2). As in UUO, the concentrating defect following relief of BUO is due, in part, to an ADH resistance of the CCT.

Segmental proximal nephron function, as reflected by rates of fluid reabsorption, is summarized in Fig. 1. In this series of studies we could detect no difference in intrinsic transport function between tubules from UUO and BUO kidneys immediately after release of obstruction. Obstruction brings about tubular functional alterations in the JMPCT and PST while ap- 
parently not affecting the SPCT. These findings are consistant with all published surface and papillary micropuncture data and provides direct information on the effect of obstruction on tubular function independent of regional renal blood flow, physical factors, or circulating natriuretic substances with a persistent in vitro effect. Segmental distal nephron function is summarized in Fig. 2. As in the proximal nephron we could detect no difference in intrinsic transport function between tubules from UUO and BUO kidneys. Obstruction alters T-ALH sodium chloride transport profoundly and imparts a state of $\mathrm{ADH}$ resistance on the CCT. The fact that tubules from UUO and BUO kidneys respond in exactly the same manner to obstruction suggests that the postobstructive diuresis observed following relief of BUO is due for the most part to changes in body composition or volume. Circulating natriuretic factors could contribute to the postobstructive diuresis following relief of BUO but it is necessary to postulate that these substances have effects only while present in situ. In our in vitro experimental setting there is no evidence for circulating natriuretic factors that may contribute to the postobstructive diuresis following relief of BUO by inducing persistent changes in tubular transport parameters.

The mechanism by which obstruction brings about changes in segmental nephron transport is unclear. Studies have shown that renal blood flow increases immediately after UUO or BUO but then progressively declines to less than half normal by $24 \mathrm{~h}$ (26). In both, UUO and BUO, medullary plasma flow decreases after ureteral obstruction and after $24 \mathrm{~h}$ is $<20 \%$ of normal flow (27). After $24 \mathrm{~h}$ of UUO and BUO in the rat, Harris and Yarger (8) using the modified Hansen technique concluded that outer cortical peritubular plasma flow is much better preserved than peritubular flow in the juxtamedullary cortex or outer medulla. The effect of acute ischemia on tubular function has recently been investigated (23). In the proximal nephron reabsorption rates are decreased, and in the distal nephron T-ALH function is reduced, and the response of the CCT to ADH is blunted. These changes are consistent with the observed alterations in tubular function found in the present study. The unique preservation of SPCT function following release of obstruction may be due, in part, to better maintenance of superficial cortical blood flow.

In summary. we have examined a rabbit model of UUO and BUO by clearance and microperfusion techniques. Analysis of segmental nephron function has shown that the intrinsic tubular effects induced by obstruction are identical in UUO and BUO. The natriuresis noted in UUO and BUO is due, in part, to disordered JMPCT, PST, and T-ALH sodium chloride reabsorption while the impaired concentrating ability is due, in part, to depressed function in T-ALH and ADH resistance of the CCT. One site for this ADH resistance of the CCT occurs at a site distal to the intracellular generation of intracellular cAMP.

\section{REFERENCES}

1. Wilson, D. R. 1980. Pathophysiology of obstructive nephropathy. Kidney Int. 18: 281-292.

2. Klahr, S., J. Buerkert, and M. C. Purkerson, 1977. The kidney in obstructive nephropathy. Contrib. Nephrol. 7: 220-249.

3. Yarger, W. E., H. S. Aynedjian, and N. Bank, 1972. A micropuncture study of postobstructive diuresis in the rat. J. Clin. Invest. 51: 625-637.

4. McDougal, W. S., and F. S. Wright, 1972. Defect in proximal and distal sodium transport in postobstructive diuresis. Kidney Int. 2: 304-317.

5. Buerkert, J., M. Head, and S. Klahr, 1977. Effects of acute bilateral ureteral obstruction on deep nephron and terminal collecting duct function in the young rat. $J$. Clin. Invest. 59: 1055-1065.

6. Sonnenberg, H., and D. R. Wilson. 1976. The role of the medullary collecting ducts in postobstructive diuresis. J. Clin. Invest. 57: 1564-1574.

7. Buerkert, J., M. L. Purkerson, and S. Klahr, 1976. On the site of decreased $\mathrm{Na}$ fluid reabsorption after release of ureteral obstruction in the rat. J. Lab. Clin. Med. 87: 397-410.

8. Harris, R. H., and W. E. Yarger, 1974. Renal function after release of unilateral ureteral obstruction in rats. Am. J. Physiol. 277: 806-815.

9. Buerkert, J., D. Martin, M. Head, J. Prasad, and S. Klahr. 1978. Deep nephron function after release of acute unilateral obstruction in the young rat. J. Clin. Invest. 62: 1228-1239.

10. Buerkert, J., D. Martin, and M. Head, 1979. Effect of acute ureteral obstruction on terminal collecting duct function in the weanling rat. Am. J. Physiol. 236: F260F267.

11. Burg, M. B., and J. Orloff, 1968. Control of fluid absorption in the renal proximal tubule. J. Clin. Invest. 47: 2016-2024.

12. Gross, J. B., M. Imai, and J. P. Kokko, 1975. A functional comparison of the cortical collecting tubule and the distal convoluted tubule. J. Clin. Invest. 55: 1284-1294.

13. Nagle, R. B., R. E. Bulger, R. E. Cutler, H. R. Jervis, and E. P. Benditt. 1973. Unilateral obstructive nephropathy in the rabbit. Lab. Invest. 28: 456-467.

14. Burg, M. B., 1972. Perfusion of isolated renal tubules. Yale J. Biol. Med. 45: 321-326.

15. Hanley, M. J., and J. P. Kokko, 1978. Study of chloride transport across the rabbit cortical collecting tubule. $J$. Clin. Invest. 62: 39-44.

16. Kawamura, S., M. Imai, D. W. Seldin, and J. P. Kokko, 1975. Characteristics of salt and water transport in superficial and juxtamedullary straight segments of proximal tubules. J. Clin. Invest. 55: 1269-1277.

17. Al-Zahid, G., J. A. Schafer, S. L. Troutman, and T. E. Andreoli. 1977. Effect of antidiuretic hormone on water and solute permeation, and the activation energies for these processes in mammalian cortical collecting tubules. J. Membr. Biol. 31: 103-129. 
18. Burg, M. B., and N. Green. 1973. Function of the thick ascending limb of Henle's loop. Am. J. Physiol. 224: 659-668.

19. Kokko, J. P. 1970. Sodium chloride and water transport in the descending limb of Henle. J. Clin. Invest. 49: 1838-1846.

20. Ramsay, J. A., R. H. J. Brown, and P. C. Croghan, 1955. Electrometric titration of chloride in small volumes. $J$. Exp. Biol. 32: 822-829.

21. Hall, D. A., L. D. Barnes, and T. P. Dousa. 1977. Cyclic AMP in action of antidiuretic hormone: effects of exogenous cyclic AMP and its new analogue. Am. J. Physiol. 232(4): F368-F376.

22. Hanley, M. J. 1980. Isolated nephron segments in a rabbit model of ischemic acute renal failure. Am. J. Physiol. 239: F17-F23.

23. Schwartz, G. J., and M. B. Burg, 1978. Mineralocorticoid effects on cation transport by cortical collecting tubules in vitro. Am. J. Physiol. 235(6): F576-F585.

24. Helman, S. I., J. J. Grantham, and M. B. Burg, 1971. Effect of vasopressin on electrical resistance of renal cortical collecting tubules. Am. J. Physiol. 220: 18251832.

25. Fine, L. G., D. Schlondorff, W. Trizna, R. M. Gilbert, and N. S. Bricker, 1978. Functional profile of the isolated uremic nephron. J. Clin. Invest. 61: 1519-1527.

26. Moody, T. W., E. Vaughan, Jr., and J. T. Gillenwater. 1977. Comparison of the renal hemodynamic response to unilateral and bilateral ureteral occlusion. Invest. Urol. 14: 455-459.

27. Solez, K., S. Ponchak, R. A. Buono, N. Vernon, P. M. Finer, M. Miller, and R. H. Heptinstall. 1976. Inner medullary plasma flow in the kidney with ureteral obstruction. Am. J. Physiol. 231: 1315-1321. 